\title{
Evaluation of indoor air quality based on qualitative, quantitative and olfactory analysis
}

\author{
YI Qin, LIU JieMin*, WANG GuiHua \& ZHANG YanNi \\ Department of Chemistry, School of Chemical and Biological Engineering, University of Science \& Technology Beijing, Beijing 100083, China
}

Received July 7, 2012; accepted August 24, 2012

\begin{abstract}
In studying indoor atmospheric pollution, the concentration of air pollutants is considered to be the primary factor in judging indoor pollution level, while sensory effects accessed by olfactory analysis has not been paid enough attention. In this paper, twenty living rooms in Beijing including newly decorated, 3 months and 6 months after decoration were sampled once a day for $10 \mathrm{~d}$, and qualitative analysis, quantitative analysis and olfactory analysis of volatile organic compounds were carried out. The results showed the concentrations of the 6 main compounds surpassed the limitation values released by World Health Organization; the pollutants with highest chemical concentrations were not the most odor active odorants. Olfactory analysis which measured the odor characters such as odor detection threshold (ODT), odor active value (OAV) and odor quality was a helpful tool to identify possible chemicals which cause indoor smelling issues, and it was necessary to access indoor air quality in combination with their chemical concentrations to give a comprehensive judgment on indoor air quality.
\end{abstract}

indoor air pollution, chemical concentration, odor detection threshold (ODT), odor active value (OAV), odor quality

Citation: Yi Q, Liu J M, Wang G H, et al. Evaluation of indoor air quality based on qualitative, quantitative and olfactory analysis. Chin Sci Bull, 2013, 58: 986-991, doi: 10.1007/s11434-013-5671-z

In recent years, pollution caused by indoor decoration has become more and more serious. As most people spend about $90 \%$ of their time indoors [1], the influence of indoor air contamination is long-termed and consecutive. Prolonged exposure to indoor toxicants can potentially lead to a variety of health issues such as dysthesia, transient morbidity, disability, disease, and even death in extreme cases [2]. Lots of studies have been performed and great public concerns have been drawn to the indoor air contamination, and the results showed that volatile organic compounds (VOCs) are the main and ubiquitous pollutants [3].

In studying indoor VOCs, with respect of connection between pollutants and human health, the toxicities of pollutants were the principal issue [4], the chemical concentration was considered to be the primary factor in judging the intensity of indoor odor pollution [5]. Therefore, series of guidelines and regulations released in many countries were

*Corresponding author (email: liujm@ustb.edu.cn) concentrated on the concentration limitation of indoor air pollutants based on toxicities [6,7].

However, some VOCs that commonly detected indoor are associated with odor [8], among which some pollutants with concentrations even lower than the limitation can strongly impact the overall sensory impression. Odor can also cause a variety of undesirable reactions among people, ranging from annoyances to documented health issues [9]. Therefore it is necessary to investigate the odor characters of pollutants combining with the concentration.

Odor characters are tested by sensory measurement by human olfactory system. Two factors including odor active value (OAV) and odor quality are usually used in odor evaluation. $\mathrm{OAV}$, which is defined as the concentration of chemical pollutant divided by its odor detection threshold (ODT) [10], is calculated to identify possible pollutants causing malodors or to express the odor activity of one odorous compound [11]. Among odor chemicals, only those with concentrations exceeding odor thresholds (OAVs are 
higher than 1) can be perceived. Odor quality is the characteristic of the smelled odor and is reported using odor description, which provides a referencing vocabulary for odor character and reflects the hedonic tone [12]. As odorants with the same OAV but with different odor qualities may smell significantly different, it is necessary to give the odor quality in odor description. Still, characterization of odors (qualitative and quantitative) can help to identify pollution sources and play an important role in evaluating indoor air quality due to great human sensitivity to odor [13].

In this study, twenty living rooms in Beijing including newly decorated, 3 months and 6 months after decoration were sampled once a day for $10 \mathrm{~d}$. Qualitative, quantitative, and olfactory analysis of VOC pollutants were carried out. The results showed that a variety of organic odor pollutants including alkane, halogenated alkanes, alcohols, aldehydes, ketones, esters, ethers, and benzene series compounds were detected in the samples. The compounds with the highest concentrations in the samples were not the most odor active pollutants. Odor quality of a sample was complicated, which was different from any of the components or the sum of all. Olfactory analysis was helpful to give a comprehensive judgment on indoor air quality in combination with quantitative analysis.

\section{Materials and methods}

\subsection{Material and instrument}

$\beta$-Phenylethyl alcohol, isovaleric acid, methyl cyclopentanone, $\gamma$-undecanoate, $\beta$-methylindole, alkanes, aldehydes and ketones were all analytical grade reagents and purchased from J\&K SCIENTIFIC LTD; alcohols, esters and benzene series compounds were analytical grade reagents and obtained from Beijing Chemical Reagents Company.

APSG-MW tubes (a glass tube filled with multi-walled carbon nanotubes bonding on the external surface of porous silica gel particulates), prepared by our laboratory [14]; Constant flow air sampler, (Beijing Municipal Institute of Labour Protection, China); Solid Phase Micro Extraction (SPME) (Supelco, USA); 1, 5, 10, $20 \mu \mathrm{L}$ and 100-mL syringe (Agilent); thermal desorption (TD) and GC-MS (Thermo TRACE GC 2000, TRACE DSQ), (Thermo Electron Corporation, USA); dynamic olfactometer, (Stillwater, Minnesota, USA).

The desorption temperature of TD was $250^{\circ} \mathrm{C}$, and helium (purity $99.999 \%$ ) was used as carrier with a flow rate of $0.1 \mathrm{~L} / \mathrm{min}$. GC/MS was equipped with a DB-5MS capillary column $(30 \mathrm{~m}$ in length, $0.25 \mathrm{~mm}$ in diameter, with a $0.25-\mu \mathrm{m}$ thick film). Helium (purity $99.999 \%$ ) was used as carrier, with a constant flow rate of $0.9 \mathrm{~mL} / \mathrm{min}$. The injection port was maintained at $220^{\circ} \mathrm{C}$ with a $15: 1$ split ratio. The initial oven temperature was $50^{\circ} \mathrm{C}$ and kept for $10 \mathrm{~min}$, and then increased to $200^{\circ} \mathrm{C}$ at a rate of $10^{\circ} \mathrm{C} / \mathrm{min}$. The temperature of the ion source in the MS was $250^{\circ} \mathrm{C}$.

\subsection{Sampling and samples analysis}

Twenty resident domiciles in Beijing including newly decorated, 3 months and 6 months after decoration were sampled and analyzed for consecutive 10 days in August. Doors and windows were kept closed for $24 \mathrm{~h}$ before sampling. Sampling was performed by APSG-MW tubes connected with air sampling pump at room temperature, with a flow-rate of $0.5 \mathrm{~L} / \mathrm{min}$ and sampling time of $10 \mathrm{~min}$.

Samples were subsequently analyzed by GC-MS. The desorbed samples were carried out from APSG-MW tubes by carrier gas of TD after pre-heating for $2 \mathrm{~min}$ [14]. At the same time, the desorbed gas was collected with a $100-\mathrm{mL}$ syringe and the VOCs were extracted by a SPME device for 15 min. Then samples extracted in SPME were desorbed in the injection port of the GC, and the sample analysis was done by GC-MS. Compound identification was based on target ions and qualifier ions of mass spectrum, retention times and comparison with library spectra. Quantification was based on regression lines, which were calculated over a range of 6 levels of concentrations versus the corresponding abundances.

\subsection{Olfactory analysis}

The medical ethics committee of University of Science and Technology Beijing (USTB) approved the protocol prior to the start of this study. All the research was performed in accordance with the Declaration of Helsinki.

Subjects between 22 and 32 years old who do not smoke were invited to attend a screening test [15]. At the start of this session, all subjects signed informed consent. They thereupon screened with 5 standard odorous dilution liquids with certain concentrations as shown in Table 1. Their odor qualities were also described.

At first, 5 odorless tapes (size: $14 \mathrm{~cm} \times 7 \mathrm{~mm}$ ) were prepared, and the top $1 \mathrm{~cm}$ of each tapes were marked as test part, the test parts of 2 tapes were soaked in a standard stimuli liquid. The remaining 3 tapes were soaked in the odor-free liquid using the same method. When the 5 soaked tapes were presented to the subjects, they were instructed to distinguish the 2 tapes containing the odorant. Each subject tested the 5 standard odorants using the same method. The subjects who could distinguish all the 5 standard odorants correctly from other 3 odorless tapes in each trial could pass the panel screening test and perform the further study.

(1) Experimental condition. Sixteen participants composed of 8 males and 8 females were finally selected. The test was carried out in an odor-free room with a temperature of $20^{\circ} \mathrm{C}$ and a relative humidity of $35 \%$. The duration of smelling was less than $2 \mathrm{~s}$, which was considered long enough to collect information of odors [16]. To avoid the participants from fatigue smelling, there was a 2 min break between tests of each stimulus, which was considered to be long enough to allow the olfactory epithelium recovery 
Table 1 Standard odorant and standard density used for the screening test

\begin{tabular}{|c|c|c|c|c|}
\hline & Standard odorant & Structural formula & Concentration (w/w) & Odor type \\
\hline A & $\beta$-Phenylethyl Alcohol & & $10^{-4.0}$ & Flowery, floral \\
\hline B & Isovaleric acid & & $10^{-5.0}$ & Bromidrosis \\
\hline $\mathrm{C}$ & Methyl cyclopentanone & & $10^{-4.5}$ & Sweet rice crust \\
\hline $\mathrm{D}$ & $\gamma$-Undecaractone & & $10^{-4.5}$ & Fruit aroma \\
\hline $\mathrm{E}$ & $\beta$-Methylindole & & $10^{-5.0}$ & Manure smelly \\
\hline
\end{tabular}

from sensory adaptation [17]. During the break, the participants reported their results.

(2) Procedure. Triangle odor bag method [15] was used with some modification for the assessment of odor detection threshold (ODT) of single VOC: when deciding the testing order of samples with different odor strengths presented to the panel, we took into account the fact that a weak odor (higher dilution) becomes more difficult to detect after introducing a strong odor (lower dilution). A descending order of odor strength is prone to intrigue olfactory adaptation of panelists. Therefore, an ascending order presentation was used in the present study.

Three odorless bags were filled with the same volume of odor-free air until the bags were almost full and closed with silicone rubber stoppers. And then certain amount of objective substance was injected into one of the 3 bags through its label with a syringe. The injection volume should meet the required concentration, at which detection occurred by chance, i.e., sub-threshold level. The other 2 bags were filled only with odor-free air (the same volumes as the first one). The 3 bags were delivered together to a subject, and he or she was instructed to smell all the 3 bags and reported from which he or she sensed an odor. The selection was mandatory, so the participants had to pick one sample based on their own olfactory senses from three sample bags at each level.

When his or her answer was incorrect, the same procedure was carried out at the next stage in which the amount of the odor was added 3 times more than the first sample. This procedure continued until the panel gave a correct answer.

The threshold was calculated for each panelist using the following formula:

$$
\mathrm{ODT}_{A}=\frac{\log a 1+\log a 2}{2},
$$

$\mathrm{ODT}_{A}$, odor detection threshold for panelist A; $a 1$, correct maximum dilution ratio; $a 2$, incorrect minimum dilution ratio.

The mean threshold values calculated for each panelist excluding minimum and maximum values was taken as the threshold value for a group of all the panelists.

For the assessment of odor quality of VOCs presented singly with certain concentration, $2 \mu \mathrm{L}$ of analytical grade objective substance was injected into a Teflon bag filled with $10 \mathrm{~L}$ of purified air at room temperature and normal pressure. When completely volatilized, the gas mixture was diluted by dynamic olfactometer to the desired concentration (the concentration of target compound tested in the real sample). Odor participants were instructed to smell at the sniffing port and describe the odor quality and evaluated whether it was easy to perceive. There were 4 levels could be used to access the difficulty for perception: easy, relatively easy, relatively hard and hard.

\section{Results and discussion}

\subsection{VOCs detected by qualitative and quantitative analysis}

TD-GC-MS analysis was firstly carried out to determine the main VOCs in the samples. Two hundred and fourty-two VOCs were detected, which could be classified as alkanes, 
esters, aromatics, alcohols, ketones, olefins, aldehydes, halohydrocarbo, cycloalkanes, ethers and other four types as shown in Table 2. The number of chemicals in certain class and the percentage of the number of chemicals in certain class attribute ratios to the total 242 chemicals are also listed in descending order. It shows clearly that the detected indoor compounds are in very large quantity and various types. And the most abundant VOCs are alkanes, esters, aromatics, alcohols and ketones. Compared to pollutants detected in office rooms [8], there are much more alcohols, ketones and esters, which may released by finishing material and fumes from the kitchen [18].

Considering the abundance and complexity of the pollutants indoors, only 40 pollutants with the presence percentage greater than $9.5 \%$ were listed in Table 3 . The presence percentages was calculated by dividing the times that a pollutant be detected in the samples by the number of total samples collected, which was 200 , and the compounds were listed in the decreasing order of their presence percentages According to the results, the detected VOCs could be classified as alkane, halogenated alkanes, alcohols, aldehydes, ketones, esters and benzene series compounds, which were also the most abundant classes shown in Table 2. Among them, benzene, naphthalene, tetrachloroethylene, trichloroethylene has been set as indoor pollutant with health risk by World Health Organization (WHO); toluene and $m, o$-xylene are in the way to be regulated by WHO [19].

Obviously the most frequently detected compounds were toluene, $m$-xylene, benzene, ethyl benzene, butyl acetate,

Table 2 Chemical class of compounds detected and their attribute ratio

\begin{tabular}{lcc}
\hline Chemical class & $\begin{array}{c}\text { Number of chemicals } \\
\text { in certain class }\end{array}$ & $\begin{array}{c}\text { Attribute } \\
\text { ratio }(\%)^{\mathrm{a})}\end{array}$ \\
\hline Alkanes & 66 & 27.2 \\
Esters & 35 & 14.4 \\
Aromatics & 34 & 14.0 \\
Alcohols & 22 & 9.1 \\
Ketones & 19 & 7.9 \\
Olefins & 18 & 7.4 \\
Aldehydes & 16 & 6.6 \\
Halohydrocarbons & 14 & 5.8 \\
Cycloalkanes & 10 & 4.1 \\
Ethers & 7 & 2.9 \\
\multicolumn{1}{c}{ 3,5-Dihydroxybenzamide } & & 1.7 \\
1,2-Diaminopropane & & \\
6-Aminoundecane & 4 &
\end{tabular}

a) Number of the chemicals in certain type/the number of total chemicals detected (242).

Table 3 Compound detected from the samples of 20 living rooms and its detectable rate

\begin{tabular}{|c|c|c|c|c|c|}
\hline Compounds & Frequency & Detectable rate $(\%)$ & Compounds & Frequency & Detectable rate $(\%)$ \\
\hline Toluene & 184 & 92.0 & $n$-Decane & 41 & 20.5 \\
\hline$m$-Xylene & 162 & 81.0 & Cyclohexanone & 41 & 20.5 \\
\hline Benzene & 150 & 75.0 & Cyclohexane & 40 & 20.0 \\
\hline Ethyl benzene & 132 & 66.0 & Nonanal & 37 & 18.5 \\
\hline Butyl acetate & 109 & 54.5 & Styrene & 35 & 17.5 \\
\hline$o$-Xylene & 108 & 54.0 & Nonane & 31 & 15.5 \\
\hline$\alpha$-Pinene & 94 & 47.0 & Decanal & 31 & 15.5 \\
\hline Tetradecane & 89 & 44.5 & Ethanol & 31 & 15.5 \\
\hline Dodecane & 84 & 42.0 & D-limonene & 30 & 15.0 \\
\hline Tridecane & 74 & 37.0 & Cyclopentane & 30 & 15.0 \\
\hline$n$-Pentadecane & 70 & 35.0 & Butane & 25 & 12.5 \\
\hline Ethyl acetate & 64 & 32.0 & Dichloroethane & 24 & 12.0 \\
\hline Hexanal & 64 & 32.0 & Tetrachloroethylene & 24 & 12.0 \\
\hline Butanol & 61 & 30.5 & 2-Ethyl-1-hexanol & 23 & 11.5 \\
\hline Hendecane & 59 & 29.5 & Trichloroethylene & 22 & 11.0 \\
\hline Pentane & 58 & 29.0 & Octyl aldehyde & 22 & 11.0 \\
\hline Hexane & 51 & 25.5 & Butyraldehyde & 20 & 10.0 \\
\hline Hexadecane & 49 & 24.5 & Isopropanol & 19 & 9.5 \\
\hline 1,2,3-Trimethylbenzene & 48 & 24.0 & Pentanol & 19 & 9.5 \\
\hline Dichlorobenzene & 44 & 22.0 & Hendecanal & 19 & 9.5 \\
\hline
\end{tabular}


and $o$-xylene as their detectable rates were much greater than $50 \%$. Most of them are ubiquitous VOCs in indoor air measured in European and North American field except for butyl acetate [3]. The 6 compounds with the highest mean chemical concentrations were listed in Table 4 . The results showed toluene was the most abundant VOC in the indoor air, with a mean value of $0.34 \mathrm{mg} / \mathrm{m}^{3}$; the concentrations of $m, o$-xylene was $0.32 \mathrm{mg} / \mathrm{m}^{3}$, and the rest ranged from 0.040 to $0.090 \mathrm{mg} / \mathrm{m}^{3}$. The relative standard deviations (RSD) of the relative response factor (RRF) ranged from $1.5 \%$ to $11.6 \%$. The relative error for nine measurements (accuracy) ranged from $3.7 \%$ to $19 \%$, and the precision for seven parallel samples ranged from $1.8 \%$ to $13.6 \%$. According to the guidelines released by WHO [19], the contents of all the 6 main compounds surpassed the limit value set in the long-term guideline, which was $0.01 \mathrm{mg} / \mathrm{m}^{3}$ to prevent potential malignant effects in the airway.

\subsection{Odor characteristics of the main VOCs tested by olfactory analysis}

Odor characteristics such as odor threshold and odor quality were tested by the method described in the Section of 1.3. Odor active values were calculated by the following equation:

$$
\mathrm{OAV}=\frac{C}{\mathrm{ODT}}
$$

where $C$ is the chemical concentration of the target compound. The results were listed in Table 4.

ODTs of the 6 main VOCs ranged from 0.077 to 8.8 $\mathrm{mg} / \mathrm{m}^{3}$; while OAVs ranged from 0.0046 to 1.8 . The most odor active stimulus, $m$-xylene, with the highest $\mathrm{OAV}$ value of 1.8, was not the most abundant composition, neither the one with the lowest ODT. Therefore it was inadequate to evaluate the odor effect of VOCs only by their chemical concentrations or odor threshold. Combination of chemical analysis with olfactory analysis could provide considerably more information. It was also helpful in the management of indoor odor pollution by finding out the most odorous pollutants.
Table 4 Odor characteristics of the 6 main single VOCs

\begin{tabular}{lccc}
\hline \multicolumn{1}{c}{ Stimulus } & $\begin{array}{c}\text { Mean concentration } \\
\left(\mathrm{mg} / \mathrm{m}^{3}\right)\end{array}$ & $\begin{array}{c}\text { Odor detection } \\
\text { threshold }\left(\mathrm{mg} / \mathrm{m}^{3}\right)\end{array}$ & $\begin{array}{c}\text { Odor active } \\
\text { value }\end{array}$ \\
\hline Toluene & 0.34 & 1.3 & 0.27 \\
$\begin{array}{l}m \text {-Xylene } \\
\text { Benzene }\end{array}$ & 0.32 & 0.18 & 1.8 \\
Ethylbenzene & 0.040 & 8.8 & 0.0046 \\
Butyl acetate & 0.080 & 0.75 & 0.11 \\
$o$-Xylene & 0.090 & 0.077 & 1.2 \\
\hline
\end{tabular}

\subsection{Analysis of change in odor quality}

We also investigated correlation of measured odor qualities of the whole samples associated with the 6 main VOCs. The odor qualities of the 200 samples and the 6 main compounds whose detection frequencies were higher than $50 \%$ were measured. The perceived results were listed in Table 5.

The perceived results of the 200 indoor samples showed they were all irritating, unpleasant and easy to perceive, although the odor levels were not exactly the same. Most of the 6 main compounds were described as slightly sweet, except for ethyl benzene which was irritating.

The samples were mixtures of some compounds including the 6 main components. However, the result showed the perceived odor of the samples changed dramatically compared to those only containing one of the 6 main components: all the 200 samples smelt irritating. The odor preference reduced compared to the single substance. It was because the olfactory system recognized complex mixtures of odorants as single entities due to odor blending, but the perception of an odorant mixture is not a simple integration of the percepts of the unmixed components [20]. A mixture of odorants could elicit a novel odor percept through configural processing (i.e., perceptual odor blending) [21].

Moreover, we noticed that odors of the compounds with high concentrations such as toluene $\left(0.34 \mathrm{mg} / \mathrm{m}^{3}\right)$ and $m$ xylene $\left(0.32 \mathrm{mg} / \mathrm{m}^{3}\right)$ were difficult to be perceived. With regard to certain compounds such as benzene $\left(0.04 \mathrm{mg} / \mathrm{m}^{3}\right)$, butyl acetate $\left(0.09 \mathrm{mg} / \mathrm{m}^{3}\right)$ and ethyl benzene $\left(0.080 \mathrm{mg} / \mathrm{m}^{3}\right)$, even low concentrations could produce strong odors which

Table 5 The perceived odor character of the whole samples and the main pollutants

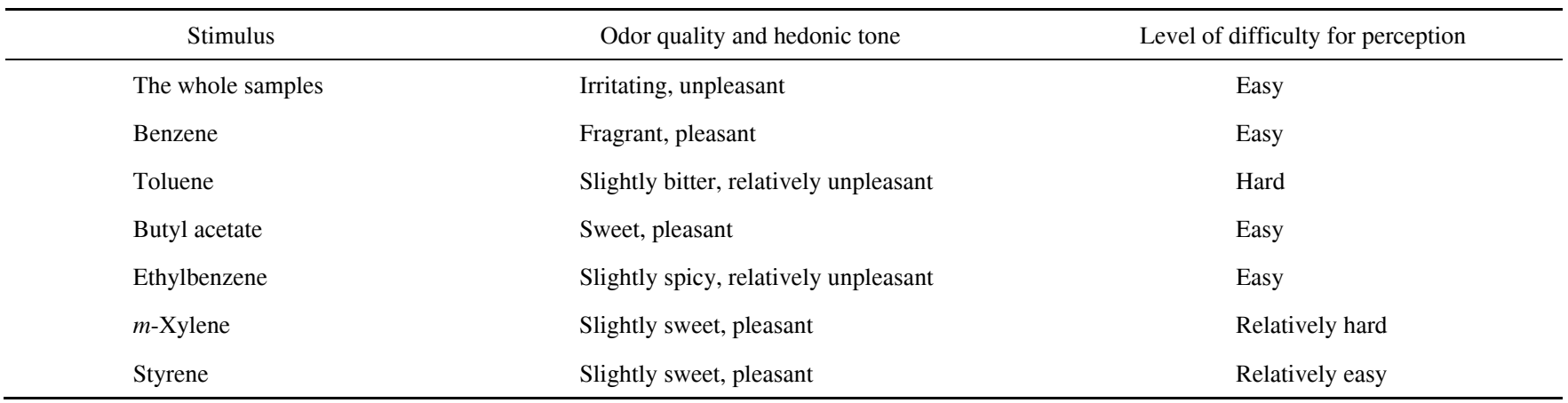


were easy to perceive. In traditional means, the hazardous effects were estimated only by judging their chemical concentrations [22]. However, compounds with low concentration could also be harmful, such as releasing irritate odor. By combining chemical analysis and olfactory tests, it was able to provide a comprehensive recognition of pollutants, and was helpful in harm prevention and control. On the other hand, the result confirmed the fact that odor quality is an important influence factor in environmental assessment when there are odorants and it is helpful in understanding which VOCs might sensory irritation.

\section{Conclusions}

The present study showed the indoor pollutants with highest chemical concentrations were not the most odor active odorants. Olfactory analysis which measured the odor characters such as ODT, OAV and odor quality was a helpful tool to identify possible chemicals which cause indoor smelling issues, and it was necessary to access indoor air quality in combination with their chemical concentrations. In addition, odor qualities of samples were complex and different from any content or the sum of all. Further investigations on multiple odor interactions are currently underway in our laboratory to unravel more details of odor quality.

This work was supported by the National High Technology Research and Development Program of China (2012AA030302), the National Natural Science foundation of China (20877008, 21277011), the Program for New Century Excellent Talents in University (NCET-09-0218), and the Fundamental Research Funds for the Central Universities (FRF-TP-10-004B, FRF-BR-11-017B).

1 Höppe P. Different aspects of assessing indoor and outdoor thermal comfort. Energ Build, 2002, 34: 661-665

2 Jones A P. Indoor air quality and health. Atmos Environ, 1999, 33: 4535-4564

3 Wolkoff P, Nielsen G D. Organic compounds in indoor air-Their relevance for perceived indoor air quality? Atmos Environ, 2001, 35: 4407-4417

4 Straalen N M V. Peer Reviewed: Ecotoxicology becomes stress ecology. Environ Sci Technol, 2003, 37: 324-330

5 Brown S K, Sim M R, Abramson M J, et al. Concentrations of volatile organic compounds in indoor air-A review. Indoor Air, 1994, 4: 123-134

6 Fanger P. Indoor air quality in the 21st century: Search for excellence. Indoor Air, 2000, 10: 68-73

7 Olesen B W, Seppanen O, Boerstra A. Criteria for the indoor environment for energy performance of buildings: A new European standard. Facilities, 2006, 24: 445-457

8 Peng $\mathrm{C} \mathrm{Y}$, Lan $\mathrm{C} \mathrm{H}$, Wu $\mathrm{T}$ J. Investigation of indoor chemical pollutants and perceived odor in an area with complaints of unpleasant odors. Build Environ, 2009, 44: 2106-2113

9 Nicell J A. Assessment and regulation of odour impacts. Atmos Environ, 2009, 43: 196-206

10 Filipy J, Rumburg B, Mount G, et al. Identification and quantification of volatile organic compounds from a dairy. Atmos Environ, 2006, 40: $1480-1494$

11 Carrapiso A I, Ventanas J, García C. Characterization of the most odor-active compounds of Iberian Ham Headspace. J Agr Food Chem, 2002, 50: 1996-2000

12 Wise P M, Olsson M J, Cain W S. Quantification of odor quality. Chem Sens, 2000, 25: 429

13 Knudsen H N, Clausen P A, Wilkins C K, et al. Sensory and chemical evaluation of odorous emissions from building products with and without linseed oil. Build Environ, 2007, 42: 4059-4067

14 Wang L, Liu J, Zhao P, et al. Novel adsorbent based on multi-walled carbon nanotubes bonding on the external surface of porous silica gel particulates for trapping volatile organic compounds. J Chromatogr A, 2010, 1217: 5741-5745

15 Iwasaki Y. The history of odor measurement in Japan and triangle odor bag method. Odor Measurem Rev, 2003, 37-47

16 Laing D G. Optimum perception of odor intensity by humans. Physiol Behav, 1985, 34: 569-574

17 Duchamp-Viret P, Duchamp A, Vigouroux M. Amplifying role of convergence in olfactory system a comparative study of receptor cell and second-order neuron sensitivities. J Neurophysiol, 1989, 61: 1085-1094

18 Landry V, Blanchet P. Vaillancourt reducing VOC emissions in the furniture and cabinets industries: An opportunity for hybrid coating systems. Bioresources, 2010, 5: 770-779

19 WHO guidelines for indoor air quality: Selected pollutants. WHO, 2010

20 Laing D G, Jinks A L. Psychophysical analysis of complex odor mixtures. Chimia Intern J Chem, 2001, 55: 413-420

21 Barkat S, Le Berre E, Coureaud G, et al. Perceptual blending in odor mixtures depends on the nature of odorants and human olfactory expertise. Chem Sens, 2011, 37: 159-166

22 Lee C W, Dai Y T, Chien C H, et al. Characteristics and health impacts of volatile organic compounds in photocopy centers. Environ Res, 2006, 100: 139-149

Open Access This article is distributed under the terms of the Creative Commons Attribution License which permits any use, distribution, and reproduction in any medium, provided the original author(s) and source are credited. 